\section{KOMPASS}

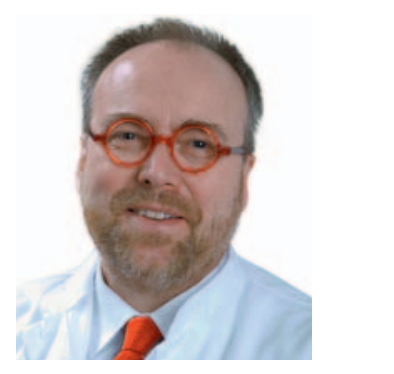

Prof. Dr. Andreas Günther ${ }^{\mathrm{BC}}$

aZentrum für Interstitielle und Seltene Lungenerkrankungen,

bUniversitätsklinikum Gießen und Marburg, Standort Gießen

Seltene Erkrankungen (orphan diseases) sind definiert als Erkrankungen mit einer Prävalenz unter 5 / 10 000. Die Versorgung dieser Patienten weist im Vergleich zu den Volkskrankheiten eine Vielzahl relevanter Schwierigkeiten und Probleme auf, die in der Konsequenz zu einer nicht unerheblichen Benachteiligung führen. Dies gilt in besonderem Umfang für seltene Lungenerkrankungen bzw. seltene Erkrankungen mit pulmonaler Beteiligung, die rein prozentual etwa 5\% aller seltenen Erkrankungen darstellen [1] und von denen in Europa geschätzt maximal 3 Millionen Patienten betroffen sind [2]. Die medizinische Versorgung von Patienten mit seltenen Lungenerkrankungen ist aufgrund folgender Faktoren in erheblichem Umfang beeinträchtigt:

\section{Eingeschränktes Wissen zum klinischen Erscheinungsbild}

Es liegt in der Natur seltener Erkrankungen, dass die involvierten Ärzte aufgrund der schieren Häufigkeit seltener Lungenerkrankungen diese vergleichsweise seltener erkennen als Volkskrankheiten. Dieses Problem könnte nur durch regelmäßige Fortbildungen im Bereich der seltenen Lungenerkrankungen überwunden werden, zum Teil auch unter Berücksichtigung interdisziplinärer Aspekte und mit Blick auf Leitsymptome, die abseits der Lungenheil-

\title{
Seltene Lungenerkrankungen - eine Herausforderung für Ärzte, Patienten und Politik
}

kunde liegen, aber einen Schlüssel zum Erkennen der Erkrankung liefern. Wer wird schon wissen, dass z.B. das pneumologische Bild einer UIP/IPF, gepaart mit einer Plättchendegranulationsstörung und/oder einem okkulo-kutanen Albinismus ein extrem starkes Indiz für das Vorliegen eines Hermansky-Pudlack-Syndroms, einer generalisierten lysosomalen Transportstörung, darstellt?

Alternativ zu regelmäßigen Fortbildungen im Bereich seltener Lungenerkrankungen erscheint es heutzutage aber auch durchaus sinnvoll, klinisch ungewohnte oder auffällige Phänotypen über Abgleich mit bestehenden Datenbanken auf das Vorliegen einer seltenen Erkrankung hin zu überprüfen. Geeignete Werkzeuge sind z.B. «The Phenomizer» (http://compbio.charite. de/phenomizer_orphanet/) von Orphanet.

\section{Limitierte Forschungsaktivitäten bei} der Aufklärung seltener Erkrankungen

Schon bei der systematischen Erfassung von Patienten mit definierten seltenen Lungenerkrankungen und der Archivierung geeigneten Biomaterials bestehen viele Hürden. Zwar existieren für einige der in Frage kommenden Erkrankungsbilder Register und Biobanken, zum Teil auch auf europäischer Ebene, dennoch ist die überwiegende Mehrzahl der seltenen Lungenerkrankungen nicht auf Register/Biobankenebene abgedeckt. Allein dies erschwert in erheblichem Umfang die systematische Erforschung dieser Erkrankungen. Noch am größten sind die Chancen der Aufdeckung der Entstehungsursachen einer seltenen Lungenerkrankung, wenn es sich um mono- genetische, vererbbare Erkrankungen handelt, d.h. wenn die Mutation in wesentlichem Umfang der Entwicklung der Erkrankung zugrunde liegt. Beispiele hierfür sind die zystische Fibrose (CFTR-Mutation), die primäre Ziliendyskinesie (z.B. DNAH5- oder DNA/1-Mutationen) oder die angeborene pulmonale Alveolarproteinose (GM-CSFRezeptor- bzw. CSF2RA-Mutation). Viele dieser seltenen, monogenetischen Lungenerkrankungen werden in ihrer Entwicklung aber aufgrund der großen Kontaktfläche der Lunge mit der Umwelt durch den potenziellen Kontakt mit putativen Noxen oder pathogenem Keimen beeinflusst, sodass die Penetranz dieser Erkrankungen oft eine gewisse Variabilität aufweist. Mit der Verfügbarkeit des «Next Generation Sequencing» (NGS) haben sich enorme Möglichkeiten aufgetan, die zu einer vollständigen Aufklärung selbst äußerst seltener Lungenerkrankungen führen können [3] und in der kommenden Dekade möglicherweise zu einer Aufklärung aller monogenetischen Erkrankungsbilder führen dürften. Allerdings ist der strukturierte Zugang zu diesen Verfahren, nicht zuletzt aufgrund der begrenzten Ressourcen im Bereich der Humangenetik, immer noch eingeschränkt und verlangsamt damit auch eine zeitnahe Aufklärung aller seltenen Lungenerkrankungen. Letztlich liegt dieser Flaschenhalseffekt nicht im zeitlichen Aufwand für die technische Durchführung der Analyse (wenige Stunden) oder in den Kosten dieser Analysen (ca. 1000 EUR) begründet, sondern in der bioinformatischen Herausforderung, solch große Datensätze möglichst korrekt und effizient zu analysieren und das verursachende Gen zu identifizieren.

\section{KARGER}

() 2017 S. Karger GmbH, Freiburg

Fax +497614520714

information@karger.com

www.karger.com
Accessible online at: www.karger.com/kkp
Prof. Dr. Andreas Günther

Zentrum für Interstitielle und Seltene Lungenerkrankungen

Universitätsklinikum Gießen und Marburg, Standort Gießen

Klinikstraße 36, 35392 Gießen, Deutschland

andreas.guenther@innere.med.uni-giessen.de 
Aber selbst, wenn entsprechende Informationen auf genetischer Ebene vorliegen, ist damit nicht zwangsläufig der Pathomechanismus einer Erkrankung aufgedeckt. Im Gegenteil, oft beginnt hier erst die aufwändige Erforschung der genaueren Krankheitsursachen, die im Zweifelsfall sehr viel mehr Zeit in Anspruch nehmen können als das NGS selbst.

Bei nicht-monogenetischen seltenen Erkrankungen ist die Aufdeckung der genetischen Varianten, die mit einer Krankheitsentwicklung oder Verschlimmerung einhergehen, schon deutlich schwieriger, da hier dann auch meist Genvarianten mehrerer Loci Einfluss auf den Verlauf der Erkrankung nehmen.

\section{Geringe Aktivitäten im Bereich der Erforschung therapeutischer Optionen}

Obschon die pharmazeutische Industrie in der Vergangenheit ihre Aktivitäten im Bereich seltener Erkrankungen deutlich ausgeweitet hat, stellt die Entwicklung pharmazeutischer Produkte für Patienten mit seltenen Erkrankungen aus mehreren Gründen eine echte Herausforderung dar:

1) Oft fehlen die entsprechenden Einsichten in die pathomechanistisch relevanten Signalwege und Zellen.

2) Der Zugriff auf patientenbasierte Biomaterialien ist meistens eher eingeschränkt.

3) In der Regel fehlen etablierte In-vitro- oder In-vivo-Modelle, um Wirkstoffbanken auf eine mögliche therapeutische Wirksamkeit hin zu untersuchen.

(4) Selbst beim Vorliegen aller genannten Punkte ist die frühe klinische Evaluierung (Phase I/II) nicht zwangsläufig vorgegeben: Klinische Prüfungen stellen aufgrund der stetig weiterentwickelten Standards und Anforderungen der Zulassungsbehörden mittlerweile eine erhebliche finanzielle Belastung dar; vor allem kleinere Unternehmen müssen angesichts von Gesamtentwicklungskosten von 100 Millionen bis einer Milliarde USD [4] im Rahmen interner Selektionsprozesse schmerzhafte Fokussierungen vornehmen. Daran ändern auch die vom Gesetzgeber vor längerer Zeit eingerichteten Erleichterungen wie «Orphan Drug Designation» im Sinne eines zehnjährigen exklusiven Vermarktungsrechtes in der Indikation oder die kostenfreie Beratung bei der Protokollerstellung zulassungsrelevanter Studien bei seltenen Erkrankungen eher wenig. Ideen zu alternativen Studienkonzepten bei seltenen oder sehr seltenen Erkrankungen existieren [5], werden aber auf Ebene der Zulassungsbehörden, mit Ausnahme einer Akzeptanz historischer Kontrollen bei sehr seltenen Erkrankungen [4], bis dato nicht berücksichtigt. Dass im positiven Fall eines Wirkungsnachweises letztlich Produkte entstehen, die Jahrestherapiekosten von fünf- (z.B. anti-fibrotische Therapie bei der IPF) bis sechsstelligen (z.B. neue Therapieverfahren bei der zystischen Fibrose) Eurobeträgen verursachen, sollte daher niemanden verwundern.

Ähnlich schwierig stellt sich die Sachlage bei der klinischen Evaluierung therapeutischer Prinzipien für Patienten mit seltenen Lungenerkrankungen durch die akademische Gemeinschaft dar. Auch hier sind durch die Einführung der «Koordinierungszentren für Klinische Studien» zwar erhebliche Fortschritte bei der Unterstützung von universitären Wissenschaftlern bei der Durchführung sogenannter Investigator Initiated Trials (IIT) erzielt worden. Dennoch bleibt die Zahl der aktuell durchgeführten Studien im Bereich seltener Lungenerkrankungen eher gering. Ähnliches gilt für die Versorgungsforschung, die durchaus auch ein erhebliches Potenzial zur Verbesserung des Behandlungsstandards bei seltenen Erkrankungen aufweist (Nationaler Aktionsplan für Menschen mit Seltenen Erkrankungen (NAMSE)), siehe auch Beitrag in «KALEIDOSKOP», S. 126 [6].

In einigen Punkten sind zuletzt aber auch Veränderungen erfolgt, die zu einer besseren Versorgung von Patienten mit seltenen Lungenerkrankungen führen können. Als Beispiel sei hier die jüngste Initiative der EU zur Einrichtung von «Exzellenz-Netzwerken zur Versorgung von Patienten mit seltenen Erkrankungen» erwähnt, in deren Rahmen auch die seltenen Lungenerkrankungen berücksichtigt worden sind. Das mittlerweile als eines der 23 solcher Exzellenz-Netzwerke (ERN) in 12/2016 von der EU akzeptierte ERN-LUNG (www.ern-lung.eu) wird sich um die Harmonisierung und Verbesserung diagnostischer Standards und eine verbesserte Kommunikation zwischen den einzelnen Referenzzentren und den Selbsthilfeorganisationen einerseits und der Öffentlichkeit/ Politik andererseits bemühen. In Deutschland ist mit der Einrichtung des Deutschen Zentrums für Lungenforschung (DZL; www.dzl.de) zudem ein weiteres, vom BMBF gefördertes Netzwerk fünf großer Lungenzentren in Deutschland entstanden, das neben Volkskrankheiten wie COPD, Lungenkrebs und Asthma bronchiale auch seltene Lungenerkrankungen wie z.B. die verschiedenen Formen diffus parenchymatöser Lungenerkrankungen (DPLD), Lungenhochdruck, ARDS, oder die zystische Fibrose adressiert. Neben der translationalen Erforschung dieser Erkrankungen werden im DZL derzeit auch übergeordnete Data-Warehouse-Strukturen implementiert, die mittelfristig eine Zusammenführung phänotypisierender Daten verschiedenster, auch seltener Lungenerkrankungen, bildgebender Daten und Ergebnisse von Analysen von Biomaterialien erzielen werden. Zudem unterstützt das DZL auch die Durchführung von frühen, Untersucher-initiierten, klinischen Studien (Phase I/II) auch im Bereich der seltenen Lungenerkrankungen.

In der Summe also scheinen sich die Rahmenbedingungen zur Versorgung von Patienten mit seltenen Lungenerkrankungen langsam zu verbessern. In diesem Sinne hoffen wir, mit der hier getroffenen Auswahl an Artikeln zu seltenen Lungenerkrankungen ein wenig zu einem besseren Bewusstsein gegenüber diesem Themenfeld beigetragen zu haben. Genießen Sie die Lektüre und denken sie an die «Seltenen» in der täglichen Routine!

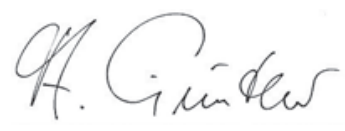

Prof. Dr. Andreas Günther 


\section{Literatur}

1 Harari S: Adding new perspectives to the European Respiratory Review. Eur Respir Rev 2016;25:101-103.

2 Spagnolo P, du Bois RM, Cottin V: Rare lung disease and orphan drug development. Lancet Respir Med 2013;1:479-487.

3 Boycott KM, Vanstone MR, Bulman DE, MacKenzie AE: Rare-disease genetics in the era of next-generation sequencing: discovery to translation. Nat Rev Genet 2013;14:681-691.

4 Institute of Medicine (US) Committee on Accelerating Rare Diseases Research and Orphan Product Development; Field MJ, Boat TF (eds): Rare diseases and orphan products: accelerating research and development. Washington D.C., National Academies Press, 2010.
5 Abrahamyan L, Feldman BM, Tomlinson G, et al.: Alternative designs for clinical trials in rare diseases. Am J Med Genet C Semin Med Genet 2016; 172:313-331.

6 Nationales Aktionsbündnis für Menschen mit Seltenen Erkrankungen (NAMSE): Nationaler Aktionsplan für Menschen mit Sseltenen Erkrankungen. Handlungsfelder, Empfehlungen und Maßnahmenvorschläge. www.namse.de/images/stories/Dokumente/nationaler_aktionsplan.pdf ( $\mathrm{Zu}$ griff 21.04.2017).

\section{FOKUS}

\section{Seltene Lungenerkrankungen}

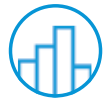

\section{Epidemiologie}

Eine Erkrankung gilt als selten, wenn die Erkrankungsrate unter 5 von 10000 Menschen rangiert. Seltene Lungenerkrankungen stellen etwa 5\% der etwa 6000 seltenen Erkrankungen dar, hierzu zählen Erkrankungen wie beispielsweise Mukoviszidose, alle Formen diffus parenchymatöser Lungenerkrankungen oder pulmonale Hypertonie. In Europa leben geschätzt 3 Millionen Patienten mit seltenen Lungenerkrankungen.

\section{Herausforderungen und Handlungsbedarf}

Seltene Krankheiten sind entsprechend wenig erforscht und stellen die Diagnostik und Therapie vor große Herausforderungen. Als Folge fühlen sich Patienten in ihrem jeweiligen Gesundheitssystem oft allein gelassen und «verwaist» (orphan disease). Patientenorganisationen kommt eine Schlüsselrolle bei der Unterstützung dieser Patienten zu (z.B. EURORDIS). Aus medizinischer Sicht gilt es, den Zeitraum bis zur Diagnose zu verkürzen und die Kenntnis der wichtigsten Merkmale von seltenen Erkrankungen bei Gesundheitsfachkräften zu vertiefen. Mit Blick auf die Arzneimittelhersteller wäre es wünschenswert, wenn für mehr seltene Erkrankungen medikamentöse Verfahren entwickelt und überprüft würden. Insgesamt gilt es, das erlangte Wissen über Datenbanken und Verzeichnisse zu verschalten, praxistauglich aufzubereiten und über Kompetenznetzwerke zur Verfügung zu stellen (z.B. Orphanet).

\section{Optionen zur Verbesserung der Versorgung}

Das Krankheitsmanagement erfordert ein interdisziplinäres und individuell abgestimmtes Vorgehen, mit dem Wissen aus Pneumologie, Endoskopie, Radiologie, Thoraxchirurgie und Pathologie. Dieses könnte durch die jüngste Ausschreibung der European Reference Networks (ERN) durch die EU erreicht werden, in deren Rahmen mittlerweile auch ein ERN-Lung (www.ern-lung.eu) akzeptiert wurde. Weitere Aktionen auf der europäischen politischen Ebene sind der Website www.rd-action.eu zu entnehmen, und sie dokumentieren, dass die EU das Thema seltene Erkrankungen sehr ernst nimmt und bemüht ist, die Versorgungslage dieser Patienten substanziell zu verbessern.

Prof. Dr. Andreas Günther, Chefarzt, Zentrum für Interstitielle und Seltene Lungenerkrankungen, Universitätsklinikum Gießen und Marburg, Gießen; Agaplesion Pneumologische Klinik Waldhof-Elgershausen, Greifenstein 